\title{
Questes
}

vestes Revue pluridisciplinaire d'études médiévales

$34 \mid 2016$

L'hiver

\section{L'hiver : introduction}

Anne Kucab et Elodie Pinel

\section{(2) OpenEdition \\ Journals}

\section{Édition électronique}

URL : http://journals.openedition.org/questes/4376

DOI : 10.4000/questes.4376

ISSN : 2109-9472

\section{Éditeur}

Les Amis de Questes

\section{Édition imprimée}

Date de publication : 15 décembre 2016

Pagination : 15-32

ISSN : 2102-7188

\section{Référence électronique}

Anne Kucab et Elodie Pinel, « L'hiver : introduction », Questes [En ligne], 34 | 2016, mis en ligne le 21 décembre 2016, consulté le 19 avril 2019. URL : http://journals.openedition.org/questes/4376 ; DOI : 10.4000/questes.4376 


\section{Introduction}

\section{Anne KuCAB et Élodie PINEL}

Université Paris-Sorbonne et Université Paris-Ouest-Nanterre-La Défense

Après «Finir le Moyen Âge », Questes s'intéresse à «L'hiver au Moyen Âge », thème dont l'intitulé fait écho au titre original du célèbre ouvrage de Jan Huizinga, L'Automne du Moyen Âge $e^{1}$. Derrière leur apparente disparité, ces deux thèmes présentent plusieurs points communs.

On y trouve d'abord le sème de la fin : l'hiver est topiquement associé à la fin d'un cycle, à ce qui disparaît pour pouvoir renaître. De même, le Moyen Âge est, par idée reçue, associé à un long entre-deux préparant une reviviscence, une résurrection, et il serait à ce titre synonyme d'une lente agonie dont on ne sait quand elle commence ni quand elle finit. Ce qui lie nos deux thèmes c'est ensuite le rapport au temps et au besoin humain d'en maîtriser le cours, d'en apprivoiser le passage, par des découpages en catégories. Dans le cas de la périodisation historique, la remise en question d'une rupture franche entre ce que certains ont, dès le XVI siècle, qualifié d' «âge moyen », et une nouvelle ère, rétrospectivement consacrée au $\mathrm{XIX}^{\mathrm{e}}$ siècle par le beau titre de « renaissance », révèle que la lecture linéaire et progressiste de l'Histoire n'est peut-être plus celle qui nous satisfait le mieux. Dans le cas de l'hiver, le rapport au temps s'ancre à la fois dans cette optique cyclique et dans une perspective linéaire: une saison, c'est un temps qui toujours

\footnotetext{
${ }^{1}$ Johan Huizinga, L'Automne du Moyen Âge, trad. Julia Bastin, Paris, Payot, 2002
} [1975]. 
recommence, qui toujours revient pour clore une période; la saison hivernale, c'est le temps qui permet, par son apparente force de destruction, d'en ouvrir un autre. Hiver aux deux visages, sa symbolique ambivalente est fixée dès la mythologie grecque où de la présence ou l'absence de Proserpine, enlevée plusieurs mois par an aux Enfers, dépend la fertilité ou l'infertilité de la nature décidée par sa mère Cérès; mythologie où aussi le dieu Janus, mi-vieil homme barbu, mi-jeune fille, règne sur le mois de Janvier qui tire de lui son nom. Temps cyclique, temps linéaire : l'hiver, comme le Moyen Âge, interroge notre rapport au temps.

Ce qui constitue la spécificité de notre thème, c'est que l'hiver s'ancre, depuis quelques décennies, dans un imaginaire plus inquiétant et que sa symbolique accuse une évolution négative. La disparition apparente de l'hiver fait naître notre malaise : le climat se réchauffe, la presse grand public parle à l'occasion d'« apocalypse climatique ». Notre désir prométhéen de progrès technologique serait puni d'un châtiment climatique : il ne nous reste qu'à expier et à soupirer, avec Villon, «mais où sont les neiges d'antan?».

La symbolique de l'hiver a-t-elle évolué négativement depuis le Moyen Âge? Sur le plan du changement climatique, il semblerait que non : la définition du climat comme prophète d'une fin du monde semble déjà valoir au Moyen Âge, à lire Eustache Deschamps : «le genrre en toute nature / Diminüent et les saisons, / Toute chose se desnature ${ }^{2} »$. Mais la négativité de l'hiver semble très forte aujourd'hui : l'apparition récente du thème de l'hiver comme objet d'étude s'inscrit sous le signe de la rigueur et de l'austérité ; la première publication y étant consacrée, à

\footnotetext{
${ }^{2}$ Eustache Deschamps, « Balade de la grant mutacion des temps et abrevacion de toute nature et approuchement de fin de monde » dans Anthologie, éd. Clothilde Dauphant, Paris, Le Livre de poche, 2014, p. 700.
} 
mettre au crédit de Martin de La Sourdière, s'intéresse à l'hiver comme à une « morte saison ${ }^{3} »$. Dans la mesure où le Moyen Âge est une période historique dont notre culture tire en grande partie sa source, on peut se demander à quoi y ressemblait l'hiver ? Comment il reçoit la symbolique antique, ambivalente, de l'hiver? Est-ce dès le Moyen Âge que cette symbolique se dégrade et tend vers la seule négativité ?

\section{L'hiver des historiens ?}

L'hiver est un thème peu étudié, l'historiographie est pauvre sur la question. En 2015 ont paru deux livres qui montrent la prise en compte et l'actualité de ce thème dans les recherches historiques ${ }^{4}$. S'il n'existe rien sur l'hiver à proprement parler, le sujet n'est pourtant pas éludé car il est inséré dans deux objets d'études qui s'y attardent fréquemment: les saisons et l'histoire du climat ${ }^{5}$.

Les saisons sont au nombre de quatre en zone tempérée ; leurs bornes correspondent aux solstices et aux équinoxes soit « une période de trois mois comprise entre un équinoxe et un solstice, dont l'alternance climatique au cours d'une année est provoquée par l'inclinaison de l'axe polaire sur le plan de l'orbite terrestre ${ }^{6} \gg$. D'un point de vue

3 Martin de La Sourdière, L'Hiver : à la recherche d'une morte saison, Lyon, La Manufacture, 1987.

${ }^{4}$ Laurent Litzenburger, Une Ville face au climat : Metz à la fin du Moyen Âge 14001530, Nancy, PUN/Éditions universitaires de Lorraine, 2015 ; Claire Judde de Larivière, La Révolte des boules de neige: Murano face à Venise, 1511, Paris, Fayard, 2014.

5 Rappelons que par climat il faut entendre selon l'OMM (Organisation Météorologique Mondiale): l'ensemble des conditions météorologiques moyennes (température, précipitations, ensoleillement, vitesse des vents...) sur une région donnée pour une période longue (au minimum tente ans). L'histoire du climat à laquelle Emmanuel Leroy Ladurie a donné ses lettres de noblesses devient un objet d'étude historique à partir des années 1960 avec la parution de plusieurs ouvrages en France et en Angleterre. Nous reviendrons sur la place que tient l'hiver dans cette approche.

Entrée «saison» du dictionnaire trésor de la langue française: 
météorologique, une saison est une époque de l'année caractérisée par un « climat » constant et un certain état de la végétation.

L'hiver est l'une de ces quatre saisons. Aujourd'hui il commence au solstice d'hiver et se termine à l'équinoxe de printemps. Au Moyen Âge, l'hiver est une saison aux contours mouvants dont les bornes dépendent des points de vue.

Initialement en effet, l'hiver fait partie d'un cycle tripartite hérité de l'Antiquité : le cycle des Heures. Cette division des Heures évolue vers un cycle à douze temps qui fait écho aux douze divisions du calendrier lunaire, et que l'on retrouve dans les enluminures des livres d'heures médiévaux. Ils coïncident progressivement avec les douze mois de l'année. Au Moyen Âge on s'éloigne du modèle tripartite (trois fois quatre mois) des Heures antiques pour adopter un schéma quadripartite (quatre fois trois mois $^{7}$ ). Ce nouveau schéma est dû aux astronomes médiévaux qui se penchent à la suite d'Aristote sur la question des météores, identifiant ainsi quatre saisons. C'est la naissance du modèle des douze mois de l'année répartis en quatre saisons. Quant à ceux qui travaillent la terre, ils distinguent plutôt, intuitivement, une saison chaude et une saison froide, limitant le nombre de saisons à deux. Dans ce schéma, le « long hiver » s'étend de novembre à février. Nous verrons dans ce bulletin que ce schéma bipartite se retrouve à plusieurs reprises dans la perception de l'hiver au Moyen Âge.

D'un point de vue culturel et religieux, l'hiver est une saison ambivalente: c'est la période des revenants et des charivaris, de la Toussaint et du Carnaval. À l'extrême négatif, le solstice d'hiver, est associé l'extrême positif, la naissance du sauveur de l'humanité, en dépit 
même de toute vraisemblance : en 532, le 25 décembre est choisi comme date pour la naissance du Christ par l'empereur Justinien alors même que les récits évangéliques, par leur mention de pâturage broutant dans les plaines de Bethléem, évoquent davantage une naissance estivale qu'hivernale $^{8}$. S'inscrivant dans cette logique des extrêmes, l'hiver s'achève avec la mort du Christ, à Pâques, dont la date doit être calculée chaque année car dépend du calendrier solaire

Quelles que soient les bornes choisies, l'hiver reste, dans l'imaginaire collectif, une saison froide et enneigée. Il est donc intéressant pour le chercheur de confronter cet imaginaire à ses sources. Quelle est la réalité du climat hivernal au Moyen Âge ?

Le perfectionnement des méthodes scientifiques a permis aux chercheurs, notamment les climatologues et les archéologues, d'avoir une vision de plus en plus précise du climat des temps passés. S'appuyant notamment sur l'analyse des glaciers (leur avancée, leur recul), les cernes de croissance du bois (dendrochronologie), l'étude des pollens (palynologie) ou encore en analysant la composition isotopique de l'oxygène contenu dans la cellulose de chêne ${ }^{9}$, il a été possible de déterminer des fluctuations et des environnements climatiques. C'est ainsi que sont apparus les notions de Petit Optimum Médiéval (POM) pour le XIII ${ }^{\mathrm{e}}$ siècle, période caractérisée par des étés chauds et des températures annuelles clémentes, et de Petit Âge Glaciaire (PAG) pour la période allant du XIV ${ }^{\mathrm{e}}$ au milieu du XIX ${ }^{\mathrm{e}}$ siècle $^{10}$. Le Petit Âge Glaciaire se caractérise quant à lui par l'avancée importante des glaciers et par des

\footnotetext{
${ }^{8}$ François Walter, Hiver : Histoire d'une saison, op. cit.

${ }^{9}$ Emmanuel Le Roy Ladurie, Histoire du climat depuis l'an mil, Paris, Flammarion, 2009 [1967] ; Des Climats et des hommes, dir. Claudie Haigneré, Jean-Paul Jacob, François Jacq, Yves Coppens, Paris, La Découverte, 2012.

${ }^{10}$ Des controverses ont porté sur une extension du POM à tout le Haut Moyen Âge.
} 
hivers froids voir neigeux ${ }^{11}$. Des éléments climatiques plus précis, comme la sécheresse et des pluies importantes, peuvent être observés en dendrochronologie.

À ces relevés scientifiques qui posent un premier cadre, l'historien doit rajouter les sources textuelles qui permettent de surcroît de connaître la perception des contemporains sur ces épisodes climatiques. Cette manière de procéder, connue pour l'établissement de la date des vendanges par exemple, peut s'appliquer à l'hiver. Pierre Alexandre en a fait la démonstration en 1987 avec son ouvrage Le Climat de l'Europe au Moyen Âge $e^{12}$. À l'historien d'être attentif car les chroniques comportent de nombreuses notations sur les fleuves gelés, les températures extrêmes ou la neige. Ainsi en décembre 1421, le bourgeois de Paris relève dans son journal : « Il gelait si fort que tout Paris était pris de glace et de gelée, et ne pouvait-on moudre à nul moulin à eau nulle part qu'à ceux au vent, pour les grandes eaux ${ }^{13} »$. Cette paralysie de l'activité économique est encore perceptible en 1432 :

Le 13 e jour de janvier, après l'allée du roi proprement, gela si âprement 17 jours ensuivants que [Seine], qui était très grande, comme jusque dedans la Mortellerie, fut toute prise de la gelée jusqu'à Corbeil, $[\ldots]$ Et si disaient les mariniers qu'elle avait plus de 2 pieds d'épais [environ $0,70 \mathrm{~m}$ d'épaisseur], et bien y apparaissait, car on allait par-dessus, on y charpentait pieux pour mettre au-devant des moulins pour rompre la glace au dégel, on y levait engins pour frapper les pieux, mais oncques ne s'en démentait. ${ }^{14}$.

\footnotetext{
${ }^{11}$ Emmanuel Le Roy Ladurie, Histoire humaine et comparée du climat, Paris, Fayard, 2004, p. 7-16.

${ }^{12}$ Pierre Alexandre, Le Climat en Europe au Moyen Âge : contribution à l'histoire des variations climatiques de 1000 à 1425, d'après les sources narratives de l'Europe occidentale, Paris, Éd. de l'École des Hautes Études en Sciences sociales, 1987.

13 Journal d'un bourgeois de Paris : de 1405 à 1449, éd. Colette Beaune, Paris, Librairie générale française, 1989, p. 175.

${ }^{14}$ Ibid., p. 310-311.
} 
D'autres sources mentionnent un froid tel que l'encre gelait dans les encriers ${ }^{15}$.

Les réalités climatiques liées à l'hiver sont donc bien présentes dans les sources, à condition d'y prêter attention ${ }^{16}$. Reste pour l'historien un point à éclaircir : comment vit-on l'hiver au Moyen Âge ?

Nombreuses sont les sources qui suggèrent des réponses. Même les sources normatives peuvent nous donner des indications, notamment sur les réactions face à un hiver particulièrement rude : c'est ainsi qu'en janvier 1482, durant un hiver difficile, Louis XI prend des mesures pour lutter contre la famine qui s'installe suite à l'hiver rigoureux de 14801481 et à l'été pluvieux de 1481 et ordonne la libre circulation des grains, l'interdiction de faire des réserves de grains et de le vendre à l'étranger. Les circonstances sont alors particulièrement dramatiques et la famine liée au froid rend les conditions de vie difficiles dans certaines régions ${ }^{17}$. D'autre part, la majorité des informations nous vient des calendriers et des livres d'heures qui illustrent abondamment les mois hivernaux. Il ne faut toutefois pas s'arrêter aux images. Hélène Dauby s'est ainsi intéressée à la nourriture en s'appuyant sur divers textes dont celui du Mesnagier de Paris $^{18}$. Il ressort de son étude que janvier est le mois où l'on récolte les panais, février le mois des poules et de la cuisson des vieilles poires, novembre le mois où l'on tue le verrat et où l'on cuisine des carottes et

\footnotetext{
${ }^{15}$ Voir aussi Jean Sarramea, «1407-1408 : un grand hiver de la fin du Moyen Âge en Europe occidentale », La Météorologie, vol. 27, 1999, p. 43-46.

${ }^{16}$ Les différentes études citées montrent en effet que les aléas climatiques sont mentionnés dans de nombreuses sources. Toutefois, ces mentions sont à manier avec précautions car elles relèvent souvent de la subjectivité de l'auteur et du contexte ; aussi est-il nécessaire de les confronter entre elles afin de bien caractériser ces épisodes climatiques et éventuellement de les classer.

17 Emmanuel Le Roy Ladurie, Histoire humaine et comparée du climat, op. cit., p. 149.

${ }^{18}$ Hélène Dauby, «Les saisons et les mets à la fin du Moyen Âge, en Angleterre et en France », dans La Ronde des saisons. Les saisons dans la littérature et la société anglaises au Moyen Âge, dir. Leo Carruthers, Presses Universitaires de Paris Sorbonne, Paris, 1998, p. 101-109.
} 
des navets et enfin, décembre, le mois où l'on tue la truie.

L'hiver est effectivement la période durant laquelle on tue le cochon, ainsi qu'en témoigne les illustrations de nombreux livres d'heures ou traités ${ }^{19}$. Ce phénomène s'explique par la naissance des cochons au printemps et leur engraissement progressif jusqu'en hiver (notamment en automne où on les représente souvent en train de manger des glands). Comme nous pouvons le lire dans le Mesnagier de Paris :

Item pour tuer le porc. On dit qu'il faut tuer les mâles au mois de novembre et les femelles en décembre. C'est leur saison au même titre que les poules ont une saison : on parle bien des gélines de février ${ }^{20}$

Par ailleurs, la Saint-Nicolas est fêtée en décembre or selon la légende de Saint-Nicolas, celui-ci redonne vie à trois enfants tués et jetés au saloir par un boucher qui cherche à vendre leur viande à ses clients en leur faisant croire qu'il s'agit de porc ${ }^{21}$. Michel Pastoureau, qui a consacré plusieurs ouvrages au cochon, explique qu'il est un des piliers de l'alimentation médiévale, notamment d'octobre à décembre, et que sa consommation cesse au carême lorsque les obligations alimentaires religieuses sont concomitantes avec la fin du lard et des salaisons ${ }^{22}$.

Soulignons pour finir que l'historien doit se montrer prudent avec les livres d'heures, dont les représentations sont très codifiées. Georges Comet prend ainsi l'exemple du Breviari d'Amor de Mastre Ermengaud

19 Barthélémy l'Anglais, Livre des propriétés des choses, vers 1350, Paris, Bibliothèque Sainte-Geneviève, ms. 1029, fol. 125v; Bréviaire dit d'Isabelle la Catholique, vers 1497, Flandres, Londres, Bristish Library, add. ms. 18851, fol. 6v et fol. 7r.

${ }^{20}$ Le Mesnagier de Paris, éd. Georgine Elizabeth Bereton et Janet Mackay Ferrier, trad. Karin Ueltschi, Paris, Librairie générale française, 1994, p. 590-591.

${ }^{21}$ Michel Pastoureau et Jean-Pierre Coffe, Le Cochon, Paris, Gallimard, 2013, p. 97.

22 Op. cit. ; Michel Pastoureau, Le Cochon : histoire d'un cousin mal aimé, Paris, Gallimard, 2009. 
écrit vers 1288 et maintes fois copié au cours des siècles suivants ${ }^{23}$. Ce livre est une sorte d'encyclopédie ; un de ses chapitres, intitulé : «De la nature des douze mois de l'année », explique comment représenter les différents mois en peinture (voir tableau ci-dessous pour l'hiver) ${ }^{24}$. Cette caractérisation des mois est mise en pratique par des vignettes illustratives dans un manuscrit du XIV ${ }^{\mathrm{e}}$ siècle conservé à la $\mathrm{BnF}^{25}$.

\begin{tabular}{|c|c|c|c|}
\hline $\begin{array}{l}\text { Vers dans } \\
\text { l'édition } \\
\text { Azais }^{26}\end{array}$ & Mois & $\begin{array}{l}\text { Caractéristique du mois (traduction } \\
\text { fondée sur celle proposée par Georges } \\
\text { Cormet) }\end{array}$ & $\begin{array}{l}\text { Eléments à représenter pour } \\
\text { figurer le mois (traduction } \\
\text { sommaire fondée sur celle } \\
\text { proposée par Georges Cormet) }\end{array}$ \\
\hline $\begin{array}{l}\text { v. 6564- } \\
6582\end{array}$ & Janvier & $\begin{array}{l}\text { «Janvier est le premier de tous / et } \\
\text { sache qu'on figure / janvier dans la } \\
\text { peinture » }\end{array}$ & $\begin{array}{l}\text { "Janvier à deux visages pour } \\
\text { montrer l'issue et l'entrée } \\
\text { On le peint aussi mangeant et } \\
\text { buvant car il fait froid et le } \\
\text { corps demande alors davantage } \\
\text { de nourriture.» }\end{array}$ \\
\hline $\begin{array}{l}\text { v. } 6583- \\
6600\end{array}$ & Février & $\begin{array}{l}\text { «Après le mois de janvier / vient le } \\
\text { mois de février / lequel est plus froid et } \\
\text { sec ? / et naturellement fort pluvieux » }\end{array}$ & $\begin{array}{l}\text { «Il est peint avec une vieille } \\
\text { figure chauffant ses pieds au feu } \\
\text { car il fait froid. » }\end{array}$ \\
\hline $\begin{array}{l}\text { v. } 6715- \\
6736\end{array}$ & Novembre & $\begin{array}{l}\text { «Sa qualité est contraire à l'humaine } \\
\text { nature, car froid et sec et par là, enlève } \\
\text { la chaleur naturelle dans le corps de } \\
\text { l'animal. » }\end{array}$ & $\begin{array}{l}\text { «Les bêtes mangent davantage. } \\
\text { On les peint dans le bocage à } \\
\text { garder les porcs et pâturent les } \\
\text { glands. » }\end{array}$ \\
\hline \begin{tabular}{|l|} 
v. $6737-$ \\
6756
\end{tabular} & Décembre & $\begin{array}{l}\text { «Décembe est dit le douzième / le } \\
\text { temps de ce mois / est naturellement le } \\
\text { grand froid / le soleil ne peut } \\
\text { descendre plus bas / Le porc est alors } \\
\text { gras » }\end{array}$ & $\begin{array}{l}\text { «Décembre est peint en } \\
\text { boucher qui frappe le porc avec } \\
\text { la cognée. » }\end{array}$ \\
\hline
\end{tabular}

Pour autant les traités agraires se détachent partiellement de cette tradition. C'est le cas du Traité d'agriculture de Pietro de' Crescenzi dont la première version datée de 1305 est perdue. Le musée Condé à Chantilly

\footnotetext{
23 Georges Comet, «Les calendriers médiévaux illustrés, supports idéologiques complexes ", dans Les Calendriers, leurs enjeux dans l'espace et le temps, dir. Jean Lefort, Jacques Le Goff, Perrine Mane, Paris, Somogy, 2002, p. 249-258.

${ }^{24}$ Maître Ermengaud, Le Breviari d'amor. 1, introduction par Gabriel Azaïs, Slatkine, Genève, 1862 , p. 359 et suivantes de la version pdf Gallica (http://gallica.bnf.fr/ark:/12148/bpt6k4247j).

${ }^{25}$ Op. cit., Lettre à sa sœur ; traduction du Salve regina ; traduction de la Légende du bois de la Croix, 1301, Paris, BnF, ms. fr. 858, fol. 48v à 52r (pages 105 à 109 de la version pdf Gallica : (http://gallica.bnf.fr/ark:/12148/btv1b6000952h).

${ }^{26}$ Op. cit., p. 359-367 de la version pdf Gallica.
} 
en possède une version de 1459 intitulé : Rustican ou Livre des prouffitz. champestres et ruraulx qui nous permet de constater que l'hiver peut être vue comme une saison de préparation des beaux jours : saison où l'on ramasse de l'argile pour faire tuiles et briques (janvier) ou celle où l'on répand le fumier dans les champs pour enrichir la terre (février) ${ }^{27}$. D'autres livres d'heures montrent fréquemment le ramassage du bois pour nourrir le feu.

L'hiver est donc bien présent dans la vie quotidienne du Moyen Âge pour autant il existe une grande différence entre l'hiver tel qu'il est vécu et tel qu'il est conté ou représenté. Dans ces derniers cas, l'hiver est constamment utilisé comme un symbole qu'il faut comprendre et décrypter.

\section{Les représentations de l'hiver}

Comment est perçu l'hiver dans l'art et la littérature ? Les premières représentations des mois d'hiver à attirer notre attention, parce que ce sont les plus topiques, sont celles des livres d'heures. En dehors des travaux ruraux de coupe du bois ou d'exécution du cochon, l'hiver y est souvent associé à la recherche du confort : les enluminures montrent à l'envi des hommes réchauffant leurs bas et leurs pieds au feu de la cheminée $^{28}$. D'autres images tirées des livres d'heures insistent sur l'hiver comme temps culinaire : ces calendriers montrent des hommes et des femmes cuisant du pain en décembre, faisant un festin en janvier,

\footnotetext{
${ }^{27}$ Pietro de' Crescenzi, Rustican ou Livre des prouffitz champestres et ruraulx, 1459, Chantilly, Musée Condé, ms. 340.

${ }^{28}$ Heures à l'usage de Rome, 1475-1499, Paris, Bibliothèque Mazarine, ms. 0502, fol. 2 ; Barthélémy l'Anglais, Livre des propriétés des choses, vers 1350, Paris, BSG, ms. 1029, fol. 124v ; Heures à l'usage de Tours-Vie de sainte Marguerite, vers 1490, Paris, Bibl. Mazarine, ms. 0507, fol. 1 ; Bréviaire dit « d'Isabelle la Catholique », vers 1497, Flandres, London, Bristish Library, add. ms. 18851, fol. 1v ; Livre d'Heures de Jeanne I de Castille, vers 1485-1506, Flandres, Londres, Bristish Library, add. ms. 18852, fol. 1v.
} 
préparant des crêpes en février ${ }^{29}$. Les protégés de Saturne, qui veille sur l'hiver, sont d'ailleurs les paysans et les boulangers ${ }^{30 .}$ L'hiver des livres d'heures est ainsi une saison représentée par des activités saisonnières plutôt positives pour les nobles, quand les vilains sont, eux, condamnés à souffrir des rigueurs du climat ${ }^{31}$.

Un changement dans les représentations artistiques de l'hiver semble intervenir à la fin du Moyen Âge. On voit se multiplier les paysages d'hiver dans les enluminures des $\mathrm{XIV}^{\mathrm{e}}$ et $\mathrm{XV}^{\mathrm{e}}$ siècles et dans les peintures flamandes $\mathrm{du} \mathrm{XVI} \mathrm{XI}^{\mathrm{e}}$ siècle ${ }^{32}$. En effet, l'hiver pendant le bas Moyen Âge est une saison rigoureuse en comparaison avec les hivers plus cléments du début et du milieu de cette période : le Petit Âge Glaciaire commence au bas Moyen Âge pour s'achever au XVIII siècle. Pourtant, ces représentations d'hivers rigoureux insistent sur l'un des phénomènes climatiques associés à l'hiver, à savoir la neige : on y voit beaucoup de paysages enneigés mais très rarement des scènes de pluies ou de tempêtes, de ciels couverts, de brumes, comme le montre Alexis Metzger pour

${ }^{29}$ Livre d'heures du XVI siècle, Oxford, Bodleian Librairy, ms. Douce 135, fol. 2v ; Livre d'heures de Jeanne I de Castille, Bruges 1486-1506, London, British Library, add ms 18852, fol. 1v. La Chandeleur est dans le monde chrétien médiéval la célébration de la purification de la Vierge et de la présentation de Jésus au Temple (au quarantième jour après la naissance conformément à la loi juive). Cette fête était au Moyen Âge l'occasion de grandes processions lumineuses où les chandelles étaient très présentes d'où l'emploi du terme de Chandeleur. Elle coïncidait avec une fête antique qui était la recherche de Proserpine enlevée par Hadès ; à cette occasion des recherches étaient effectuées à l'aide de torches et l'on consommait des galettes de céréales en hommage à sa mère (Cérès). C'est là l'origine de nos crêpes.

30 Bridget Anne Henisch, The Medieval Calendar Year, University Park, The Pennsylvania University Press, 1999, p. 34.

${ }^{31}$ Ce rapport différencié à l'hiver en fonction des classes sociales dans les livres d'heures est relevé par Wendy. Harding, "Sir Gawain and the Green Knight as a winter's tale », dans La Ronde des saisons, dir. Leo Carruthers, Paris, Presses Universitaires de la Sorbonne, 1998, p. 70. Fleur Vigneron souligne l'association de l'hiver aux vilains dans la lyrique de la fin du Moyen Âge (Fleur Vigneron, Les Saisons dans la poésie française des XIV et XV siècles, Paris, Champion, 2002, p. 350).

32 Pieter Bruegel, Le Retour des chasseurs, 1565, Kunsthistorisches Museum, Vienne, Autriche. 
l'histoire de l'art et Fleur Vigneron pour la lyrique de la fin du Moyen Âge ${ }^{33}$. Pourquoi ce choix de la neige? Servirait-il un embellissement du thème hivernal $^{34}$ ? Ou traduit-il simplement la réalité d'un enneigement plus abondant à la fin de notre période ?

Enfin, si, en art, le Moyen Âge associe, comme nous, l'hiver à une fin, il n'exclut pas pour autant le sème du commencement. Certes, l'hiver symbolise la fin d'un cycle, la fin d'une vie. L'association de l'hiver et de la vieillesse se trouve ainsi dans la mise en parallèle des mois de l'année et des âges de la vie ${ }^{35}$ : chaque mois représente six ans de vie et le mois de Décembre est associé à la fin de la vie :

Avant que vienne en Décembre Tous luy appetissent ly membre Car il a soixante douze ans En ce mois tout se meurt le temps Toute verdeur pert sa puissance Tous esbas sont en desplaisance Et tous enseignent cest la somme Quit ny a mes puissance en homme Puis qu'il a soixante douze ans Il aimeroit mieux deux chaux flans que l'amour d'une damoiselle Mol lit et patfonde escuelle Avoir est toute sa voulente Passe a maintz puer et est Et sil vault pis en l'an qu'antan Ainsi ne vit l'homme qu'un $\mathrm{an}^{36}$.

${ }^{33}$ Alexis Metzger, Plaisirs de glace : essai sur la peinture hollandaise hivernale du Siècle d'or, Paris, Hermann, 2012 ; Fleur Vigneron, Les Saisons..., op. cit., p. 369370.

34 À l'image de cette représentation de la neige tombant sur un paysage, dans Barthélémy l'Anglais, Livre des propriétés des choses, vers 1350, Paris, BSG, ms. 1029, fol. 132v.

35 «Allégorie de l'hiver» par Ambrogio Lorenzetti, Fresque du palais public de Sienne, vers 1338-1340. Cette fresque se trouve en frise du côté du mauvais gouvernement, montrant la symbolique négative de l'hiver.

${ }^{36}$ Voir le Compost ou calendrier des bergers qui possède plusieurs éditions. Nous nous appuyons notamment sur Calendrier des bergers, éd. Max Engammare, Paris, PUF, 2008. 
Ceci vaut pour autant que l'on commence l'année, selon le calendrier romain décidé par Jules César, par le mois de janvier ${ }^{37}$. Les mois de janvier et de février peuvent ainsi ne plus être associés à la vieillesse mais à l'enfance: on trouve des enluminures, dans les livres d'heures, associant l'hiver à l'enfance et l'enfance au jeu ${ }^{38}$. Saison de fêtes dans le calendrier liturgique, l'hiver devient symboliquement une saison ludique ${ }^{39}$; aux batailles de neige répondent les scènes de patinage, voire de luge $\mathrm{e}^{40}$. L'ambivalence antique de l'hiver, dont nous avons parlé tout à l'heure, se retrouve donc au Moyen Âge dans cette tension de l'hiver entre vieillesse et enfance.

Les représentations artistiques de l'hiver au Moyen Âge sont donc, pour partie, en résonance avec la réalité de cette saison ; elles conservent, d'autre part, l'ambivalence de sa symbolique, héritée de l'antiquité. À ce titre, c'est peut-être la célèbre représentation des Très riches heures $d u$ duc de Berry des frères de Limbourg qui concentre le mieux cette ambivalence hivernale dans son ensemble: froid extérieur, chaleur du foyer ; austérité de la nature endeuillée, grivoiserie (carnavalesque ?) des bas-ventres de femmes se réchauffant à l'abri ; activité au-dehors, silence et repos au-dedans ${ }^{41}$.

\footnotetext{
${ }^{37}$ D'où le double visage, de vieillard et de jeune femme, de Janus. Il s'agit d'un livre mêlant médecine, morale et astrologie, imprimé pour la première fois à Paris par Guy Marchant ; voir Bridget Anne Henisch, The Medieval Calendar Year, op. cit., p. 136-137.

${ }^{38}$ Ibid., p. 139 et 152, voir aussi la miniature du mois de janvier dans ce manuscrit conservé à Londres. (British Library, add. ms. 24098, fol. 18v).

39 Georges Comet, «Les calendriers médiévaux illustrés, supports idéologiques complexes », art. cit.; Georges Comet, «Les calendriers médiévaux, une représentation du monde », Journal des savants, 1992, vol. 1, n 1, p. 35-98.

${ }^{40}$ Livre d'Heures, vers 1485-1506, Flandres, Londres, Bristish Library, add ms. 18852, fol. 2r; Heures d'Adélaïde de Savoie, 1460-1465, Chantilly, Musée Condé, ms. 76, fol. 12r.

${ }^{41}$ Très riches heures du Duc de Berry, Frères Limbourg, 1412-1416, Chantilly, musée Condé, ms. 65, fol. 2v; Brevarium Grimani, vers 1510, Venise, Bibliothèque Marciana fol. 2v.
} 
En littérature, à première vue, la symbolique hivernale semble davantage tirer vers la négativité et l'ambivalence de cette saison semble disparaître. La lyrique des troubadours est en effet connue pour célébrer l'Été à travers la « reverdie» du mois d'avril ou les fêtes amoureuses du mois de mai, mois qui sont dans la poésie savante latine associés au printemps mais à l'été dans la poésie en langue vernaculaire ${ }^{42}$. L'hiver semble ainsi être opposé à l'été comme un temps non propice à l'amour ; ainsi chez Guillaume de Poitiers : «Je ferai chansonnette neuve / Avant qu'il vente, gèle ou pleuve ${ }^{43} »$.

Pourtant, si l'hiver symbolise les obstacles à l'amour, ces obstacles se révèlent parfois nécessaires pour éprouver la sincérité du cœur de l'ami et peuvent se parer, en ce sens, d'une certaine positivité : «Ainsi va-t-il de notre amour / Comme la branche d'aubépine / Tout au long de la nuit, tremblante / Elle endure le froid, la pluie / Le lendemain vient le soleil ${ }^{44} \gg$. Les frimas hivernaux peuvent dès lors être amoureux, et le troubadour peut dire de sa dame « pour elle j' ai froid, je tremble ${ }^{45} »$.

Le renversement des topiques troubadouresques fait revisiter la symbolique hivernale; lorsque le cœur de la dame est froid, l'hiver devient la saison où chanter : «Quand la douce brise s'aigrit, / Que les feuilles tombent des branches, / Que le babil de l'oiseau change, / Je soupire et chante l'amour ${ }^{46} »$. Certains troubadours, sûrs d'être aimés,

42 Jacques Le Goff, La Civilisation de l'Occident médiéval, Paris, GarnierFlammarion, 2008 [1964], p. 153.Dans une ballade d'un troubadour anonyme, la reine amoureuse est dite « avrileuse » et la saison qui y est associée est qualifiée de « temps joli » (Poésie des troubadours: Anthologie, éd. René Nelli, René Lavaud et Henri Gougaud, Paris, Seuil, 2009 [1966], p. 34 et p. 33).

43 «Farai chansoneta nueva / Ans que vent ni gel ni plueva», Guillaume de Poitiers, «Chanson» dans Chansons de trouvères, éd. Samuel N. Rosenberg et Hans Tischler avec la collaboration de Marie-Geneviève Grossel, Paris, Livre de poche, 1995, p. 37.

44 «La nostr'amor vai enaisi / Com la branca de l'albespi / Qu'esta subre l'arbre en treman, / La nuoit, a la ploja ez al gel / Tro l'endeman, que-1 sols s'epan », ibid., p. 39. ${ }^{45}$ Ibid., p. 39.

46 «Quant l'aura doussa s'amarzis / E-l fuelha chai de sul verjan / E l'auzelh chanjan 
attendront ainsi les rigueurs de cette saison le cœur léger :

Mon coeur est si plein de joie

Qu'il trompe Nature.

Le frimas, qu'est-il pour moi ?

Blanche fleur, jaune, vermelle.

Plus il vente, plus il pleut

Plus je suis heureux [...]

Mon coeur est tant amoureux

Tant pris de joie douce

Que gelée me semble fleur

Et neige verdure ${ }^{47}$.

« [C]omme l'expliquent abondamment les trouvères, le véritable amoureux aime en tout saison, et non pas seulement au printemps » rappelle Michel Zink ${ }^{48}$. La symbolique de l'hiver dans la lyrique des troubadours n'est pas si négative qu'il semble à première vue, ou plutôt c'est parce qu'elle est topiquement acceptée comme négative qu'elle permet un jeu de renversement du négatif et du positif, des valeurs associées à l'été et à l'hiver.

Qu'en est-il des autres facettes de la symbolique hivernale médiévale lorsqu'on aborde l'histoire littéraire? Nous avions d'abord évoqué l'hiver comme temps où l'on recherche le confort. Or, en littérature, l'hiver ne se présente pas de manière évidente comme le temps $\mathrm{du}$ repos. On trouve bien un Colin Muset se faisant l'écho des enluminures des livres d'heures dans «Quant je le tens refroidier», quasiment à la manière d'une ekphrasis ${ }^{49}$. Et on peut, à l'instar de

lor latis, / Et ieu de sai sospir e chan », Cercamon, « Chanson » dans op. cit., p. 40.

47 «Tant ai mo cor ple de joya / Tot me desnatura; / Flor blancha, vermelh'e groya / Me par la freyura / C'ab lo ven et ab la poya / E mos chans melhura [...] / Tant ai cor d'amor / De joi e de doussor / Per que-l gels me sembla flor / E la neus verdura », Bernard de Ventadour, «Chanson»dans op. cit., p. 58.

${ }^{48}$ Michel Zink, Introduction à la littérature française du Moyen Âge, Paris, Livre de poche, 1993, p. 51.

49 «Quant je le tens refroidier / Voi, et geler, / Et ces arbres despoillier / Et iverner, / Adone me vueil aisier / Et sejorner / A bon feu, lès le brasier, / Et à vin cler, / En chaude maison, / por le tens felon. / Ja n'ait il pardon / Qui n'aime sa garison! »; 
Christopher Lucken, associer à l'hiver la lecture intime, le recueillement et le silence. Pourtant, l'hiver est bien plus souvent représenté dans les romans comme un temps adverse, contre les difficultés duquel il faut se défendre, une saison qui pousse à l'activité bien plus qu'à l'oisiveté : Fleur Vigneron a relevé l'association de l'hiver aux images guerrières comme au sommeil et au manque d'inspiration du poète ${ }^{50}$. Ainsi, dans le Roman de Renart, l'hiver et l'épuisement des ressources poussent le goupil à de nouveaux forfaits : «Seignors, ce fu en cel termine / Que li doz tens d'esté define / Et yver revient en saison, / Que Renart fu en sa maison. / Sa garison a despendue ; / Ce fut mortel descovenue ${ }^{51} »$.

Autre facette de la symbolique hivernal dans l'art médiéval, l'adoucissement de la négativité de l'hiver par l'évocation de la neige se retrouve en littérature. La neige peut être l'occasion de motifs méditatifs, suspendus : ainsi de l'épisode de Perceval méditant sur Blanchefleur à qui il a promis de revenir et à laquelle il ne revient pas, frappé de stupeur face aux taches de sang dans la neige: « Li sanz et la nois ensanble / La fresche color li resanble / Qui est en la face s'amie ${ }^{52} »$. Mais elle peut, comme sur les enluminures, donner lieu à une scène plus pittoresque : ainsi des «mouches blanches» dont parle Rutebeuf dans le «Dit des

«Quand je vois le temps se refroidir/ et geler/ et les arbres se dépouiller / et l'hiver s'installer, / alors je veux me mettre à l'aise / et séjourner / près du brasier d'un bon feu, / avec du vin clair, / dans une maison chaude, / à cause du temps mauvais. / Qu'il ne connaisse jamais de pardon/ Celui qui n'apprécie pas son bien-être!» (Colin Muset, «Quant je lou tans refroidier », I dans Les Chansons de Colin Muset, éd. Joseph Bédier, Paris, Champion, 1969, p. 752-755).

${ }^{50}$ Fleur Vigneron, Les Saisons..., op. cit., p. 346-348.

51 «Messieurs, c'était l'époque où prend fin le doux temps d'été, et où revient la saison d'hiver. Renart se trouvait dans sa demeure. Toutes ses provisions étaient épuisées : quelle funeste mésaventure !» (Le Roman de Renart, 2. "Si conme Renart manja le poisson aus charretiers », v. 1-6, éd. Naomuki Fukumoto, Noboru Harano et Satoru Suzuki, révision, présentation et traduction par Gabriel Bianciotto, Paris, Livre de poche, 2005, p.134-135).

52 «[...] le sang et la neige ensemble / sont à la ressemblance de la couleur fraîche / qui est au visage de son amie » (Chrétien de Troyes, Le Conte du Graal ou le Roman de Perceval, v. 4133-4135, éd. Charles Méla, Paris, Livre de poche, 1990, p. 302-303. 
Ribauds de Grève ${ }^{53}$ ». La neige est enfin symbole de douceur et de beauté, par exemple chez le troubadour Peire d'Auvergne: «Domneis d'amor qu'en leis s'espan e creis / plens de doussor vertz e blancs com es nics ${ }^{54} \gg$.

Enfin, on note dans les enluminures une conservation de l'ambivalence hivernale héritée de l'Antiquité: tantôt associée à la vieillesse, cette saison pouvait aussi être rapprochée de l'enfance et du jeu. En littérature, l'hiver a été plus souvent personnifié sous les traits d'un vieillard, traits masculins dans les personnifications de l'Hiver chez Eustache Deschamps et Charles d'Orléans, féminins dans le Secretum Secretorum, compendium médical arabe circulant au XII ${ }^{\mathrm{e}}$ siècle, et dans les débats poétiques d'Hiver et d'Été du Xv ${ }^{\mathrm{e}}$ siècle ${ }^{55}$. Les sèmes de l'enfance et du jeu sont plus discrets : concernant les batailles de boules de neige, un poète italien, Folgore da San Gimignano du XIV ${ }^{\mathrm{e}}$ siècle, écrit dans son poème «De Gennaio » («De Janvier ») ${ }^{56}$ : «Uscir di for'alcuna volta il giorno / gittando de la neve bella e bianca / a le donzelle che staran da torno $^{57}$ ». L'hiver était pourtant, au Moyen Âge, une saison ludique et « littéraire », puisque c'était en cette saison que se tenaient le plus de veillées au cours desquelles l'on pouvait entendre raconter des romans, veillées qui tenaient plus de la performance théâtrale que de la lecture publique.

\footnotetext{
53 «Les noires mouches vos ont point, / Or vos repoinderont les blanches » (Rutebeuf, « Le Dit des ribauds de Grève » dans CEuvres complètes, éd. Michel Zink, Paris, Livre de poche, 2005, p. 214-215).

54 «Les signes de l'amour qui en elles s'épanouissent et croissent / Sont si doux, verts et blancs comme neige » (Jean-Claude Marol, La Fin'Amor : chants de troubadours (XII ${ }^{e}$ et XIII ${ }^{e}$ siècles), Paris, Le Seuil, 1998, p. 9).

${ }^{55}$ Op. cit., p. 44.

${ }^{56}$ Ibid., p. 133. La figure du bonhomme Hiver se transformera peu à peu pour adopter les traits de notre «Père Noël »; au sujet du Père Noël et de son devenir au XX $\mathrm{X}^{\mathrm{e}}$ siècle, voir Claude Levi-Strauss, «Le Père Noël supplicié » dans Les Temps modernes, vol. 77, p. 1572-1590.

${ }^{57}$ « Sortir plusieurs fois par jour / pour jeter de la neige belle et blanche / sur les demoiselles qui se tiennent aux alentours » (Bridget Anne Henisch, The Medieval Calendar Year, op. cit., p. 181).
} 
Par-delà l'apparente négativité du symbole hivernal en littérature au Moyen Âge, un bref sondage des textes nous révèle donc une réalité plus complexe : comme dans les enluminures, les évocations de l'hiver en peinture semblent marquées par une certaine ambivalence.

\section{Conclusion}

Au terme de ce panorama de la symbolique artistique et littéraire de l'hiver, des lignes de force s'imposent. Topiquement, l'hiver est associé pour nous à la vieillesse, d'abord ; à une suspension de activités, rurales, commerciales et guerrières, ensuite. Or, après un premier examen des représentations artistiques et littéraires de l'hiver, il apparaît que l'hiver n'oblige ni à mourir, ni à hiberner.

De quelle manière cette négativité hivernale s'est-elle fixée dans les représentations littéraires? Telle est la première question à laquelle répond Sarah Delale dans son article sur l'hiver dans l'œuvre de Christine de Pizan et Sung-Wook Moon en présentant l'hiver vu par Rutebeuf. Nous examinerons ensuite les réalités qui se cachent derrière le symbole avec la question de la réalité de la trêve militaire hivernale pendant la Guerre de Cent ans traitée par Christophe Furon et avec la place non négligeable de l'hiver au sein des sources urbaines analysée par Anne Kucab. Nadine Cretin retracera pour finir, dans une approche anthropologique, le lent processus de fixation des symboles hivernaux au Moyen Âge dans le folklore. 